\title{
Vorwort zur 85.-99. Auflage
}

Seit dem letzten Erscheinen dieses Werkes sind mehr als 6 Jahre vergangen, eine lange Zeit, in der die medizinische Wissenschaft der ganzen Welt große Fortschritte auf allen Gebieten gemacht hat. Wie bei den früheren Auflagen, so ist auch dieses Mal wicder versucht worden, durch sorgfältiges, umfang. reiches Zeitschriftenstudium den neuen Erkenntnissen 80 weit wie möglich Rechnung zu tragen.

Die Arbeit an dieser Auflage, die einer fast völligen Neubearbeitung gleich. kommt, war besonders gro $B$ und hat sehr viel Zeit in Anspruch genommen.

Schwer getroffen wurde das Buch durch den jähen Tod meines langjährigen, unermüdlichen Helfers Dr. Hans Schlunganum, der noch in den allerletzten Tagen des Krieges, im Februar 1945, einem Flugzeugunglück zum Opfer fiel. Sein Bruder Dr. Werner Schlungbaum hat mich bei der Bearbeitung dieser Auflage besonders auf dem Gebiete der Inneren Medizin tatkräftig unterstützt. In gleicher Weise erfuhr ich unermüdliche Hilfe durch meine Assistenten Dr. E. Buchmann und Dr. R. Tenschert. Herr Apotheker C.O. LAUBSCHAT hat mir durch sachverständige, ausgiebige pharmakologische Ratschläge, Herr Dr. R. HzMnNN, der Leiter der Bakteriolog. Abteilung des Krankenhauses Friedrichshain und Herr Dr. J. LEMBKE auf bakteriologischem Gebiete sehr zur Seite gestanden.

Der Privatgelehrte Herr C. WETZEr, hat keine Mühe gescheut, etymolo. gische Unklarheiten und Fehler auszumerzen. Die technische Assistentin Frau G. Hegenwald hat in dankenswerter Weise die Texte der Untersuchungsmethoden durchgesehen und ergänzt. Beim Lesen der Korrekturen half mir Frl. cand. med. U. KLÜNDER in ausgezeichneter Weise.

lhnen allen sage ich hierınit mei..en herzlichsten Dank.

Eine Anzahl neuer Abbildungen ist hinzugckommen, einige Abbildungen sind durch bessere ersetzt worden.

Berlin, Januar 1951. 